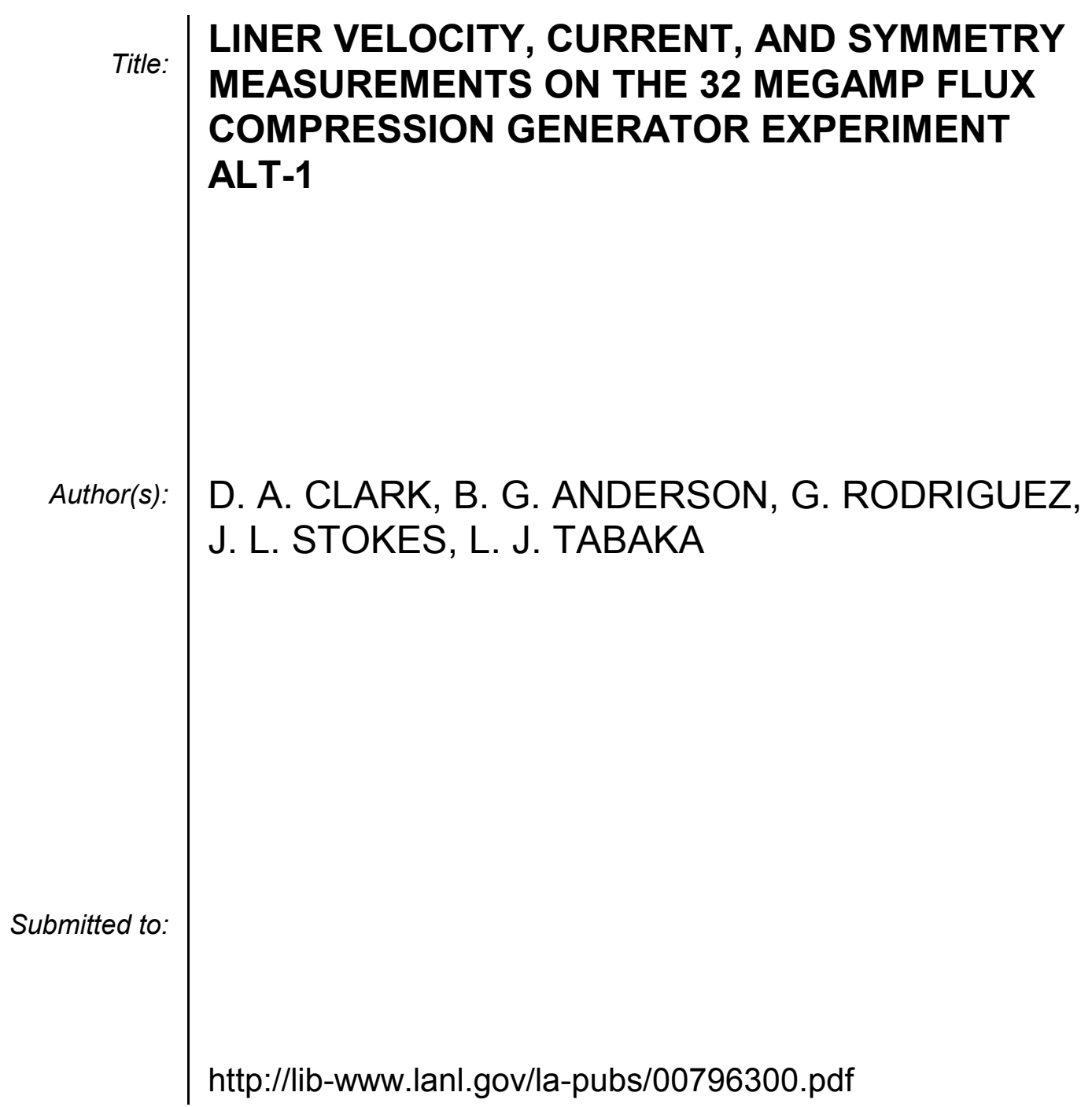




\title{
LINER VELOCITY, CURRENT, AND SYMMETRY MEASUREMENTS ON THE 32 MEGAMP FLUX COMPRESSION GENERATOR EXPERI- MENT ALT-1*
}

\author{
D. A. CLARK ${ }^{\dagger}$, B. G. ANDERSON, G. RODRIGUEZ, J. L. STOKES, L. J. TABAKA \\ Los Alamos National Laboratory, Mail Stop D410 \\ Los Alamos, NM 87544, USA
}

\section{Abstract}

A flux compression generator pulse power system, designed, built, and fielded by a Russian team at the All Russian Scientific Research Institute of Experimental Physics (VNIIEF), was used to successfully drive an aluminum liner to velocities greater than $12 \mathrm{~km} / \mathrm{sec}$. The experiment objective was to demonstrate performance of a precision liner implosion at an Atlas current of $30 \mathrm{MA}$ or greater. Diagnostics to measure liner performance were an essential part of the experiment. An experimental team from Los Alamos National Laboratory (LANL) provided a suite of diagnostics to measure liner performance. Three diagnostics were fielded: 1 . A velocity interferometer (VISAR) to continuously measure the liner innersurface velocity throughout the entire range of travel, 2 . Two Faraday rotation devices to measure liner current during the implosion, and, 3. Sixteen fiber optic impact pins to record liner impact time and provide axial and azimuthal symmetry information. All diagnostics performed very well. Major results are maximum current: $32.3 \mathrm{MA}$, velocity at impact: greater than $12 \mathrm{~km} / \mathrm{sec}$, symmetry: the impact pins indicated that the liner was smooth, solid, and axially symmetric upon arrival at the diagnostic package. The LANL team fabricated, installed, and recorded the three diagnostics presented here. All necessary equipment was brought to the site in Russia. The VNIIEF team fielded other diagnostics to measure machine performance. Results of machine diagnostics are reported in other presentations.

\section{INTRODUCTION}

The Advanced Liner Technology Experiment, ALT-1, was performed at the All Russian Scientific Research Institute of Experimental Physics (VNIIEF) in Sarov, Russia, 3 November, 1999. The purpose of the experiment was to obtain data on performance of a solid metallic imploding liner at Atlas [1] [2] [3] conditions. Figure 1 is a photograph on the ALT-1 device on the firing point. An experimental team from Los Alamos National Laboratory designed, fabricated, installed, and recorded a suite of diagnostics to measure liner performance. The VNIIEF team designed and fabricated the generator and liner.

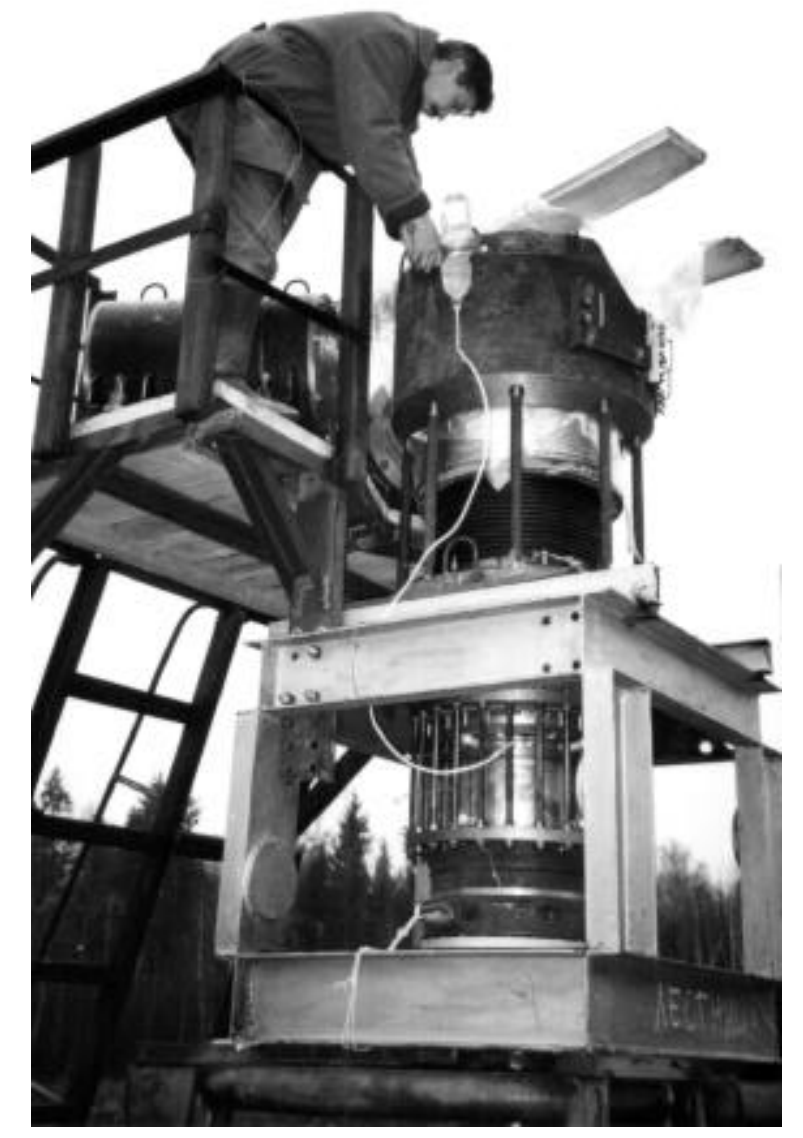

Figure 1. Photograph of the ALT-1 device on the firing point. The disk explosive electromagnetic generator (DEMG) is visible inside the heavy steel frame. Above the frame is the cylindrical transmission line, and above that is steel shielding surrounding the imploding liner experimental unit. Two radiographic x-ray sources are to the left of the shielding. Below the generator, not visible in this photograph, is the explosive driven helical electromagnetic generator (HEMG) that provides seed current for the disk generator.

\footnotetext{
* Work Supported by the Los Alamos National Laboratory under US DOE contract W-7405-ENG-36.

$\dagger$ email: daclark@lanl.gov
} 
VNIIEF implemented a suite of diagnostics, mostly inductive pickup (b-dot) probes, to record machine performance, and fielded two x-ray sources and film packs for radiographic data on the imploding liner. Results of the VNIIEF diagnostics are reported elsewhere in these proceedings [4]. Unfortunately, the radiographs were lost due to excessive damage [5].

The generator was a $400 \mathrm{~mm}$ diameter, 10 element, explosive driven disk generator with a $240 \mathrm{~mm}$ diameter explosive driven helical generator to provide 6 MA seed current. Generator output was specified to be $30 \mathrm{MA}$ with 4.0 microsecond risetime. A capacitor bank provided 100 kA seed current for the helical generator. The liner was 40 $\mathrm{mm}$ outside diameter, $2 \mathrm{~mm}$ thick, technically pure aluminum.

\section{EXPERIMENT}

The three diagnostics fielded by LANL were a velocity interferometer (VISAR)[6], two Faraday rotation current loops, and 16 fiber optic impact pins. All data presented in this paper is on a time scale that refers to one of the generator triggers, BY-19-2. Start of liner current on the BY-19-2 time scale is about 24.4 microseconds.

The VISAR probe was located along the liner axis in the Central Measuring Unit (CMU), with a 45 degree mirror installed to direct the laser beam to the liner inner surface. The CMU was a $20 \mathrm{~mm}$ diameter stainless steel cylinder, placed between the glide planes, that contained the fiber optic pins and VISAR probe. One half the length of the CMU contained the VISAR probe and mirror, and the other half contained the 16 fiber optic pins.

Figure 2 presents the VISAR velocity data and Fig. 3 presents radius vs. time derived from the velocity.

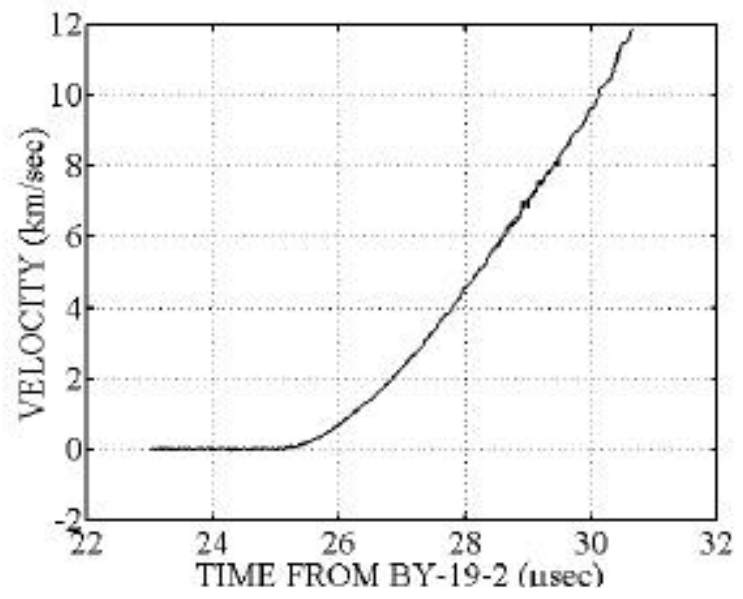

Figure 2. VISAR velocity data. The VISAR was able to track the liner inner-surface almost to the point of impact with the central measuring unit. Maximum velocity $\mathbf{e}$ corded is about $12 \mathrm{Km} / \mathrm{sec}$.

Times of liner impact from the fiber optic pins are also shown in Fig. 3 to emphasize how close to the end of travel the VISAR system was able to record high quality data.

The VISAR signal was very good throughout most of the liner travel. At the very end of travel, signal was weak due to the reflection moving out of the probe field of view. Maximum recorded velocity was greater than 12 $\mathrm{km} / \mathrm{sec}$. Accurate liner velocity data has contributed to verification of improved conductivity models [7].

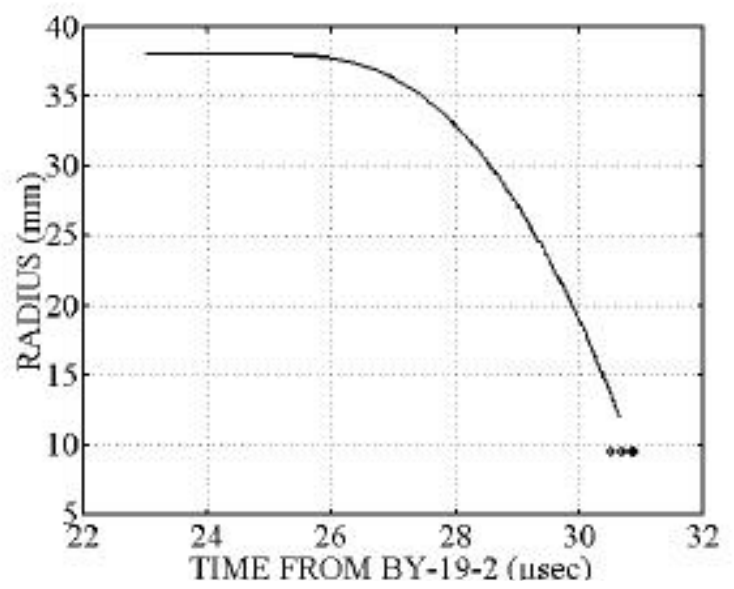

Figure 3. Radius vs. time VISAR data. Liner inner radius is the integral of the velocity data. Diamonds at the end of the RT line are the impact pin data. Pin tips were located at radius $9.5 \mathrm{~mm}$. Eight of the ten pin times are located under the right hand diamond.

For the liner current measurements, two Faraday rotation loops [8] [9], FR1 and FR2, were located in the radial transmission line between the generator output and the liner. Diameters of the two loops were $384.5 \mathrm{~mm}$ and $396.5 \mathrm{~mm}$, respectively.

Each Faraday unit consisted of a single turn of twisted fiber illuminated from one end with a diode laser, a length of polarization-maintaining fiber to transmit the signal to the quadrature splitters, the quadrature splitters themselves, and a pair of photodiode detectors.

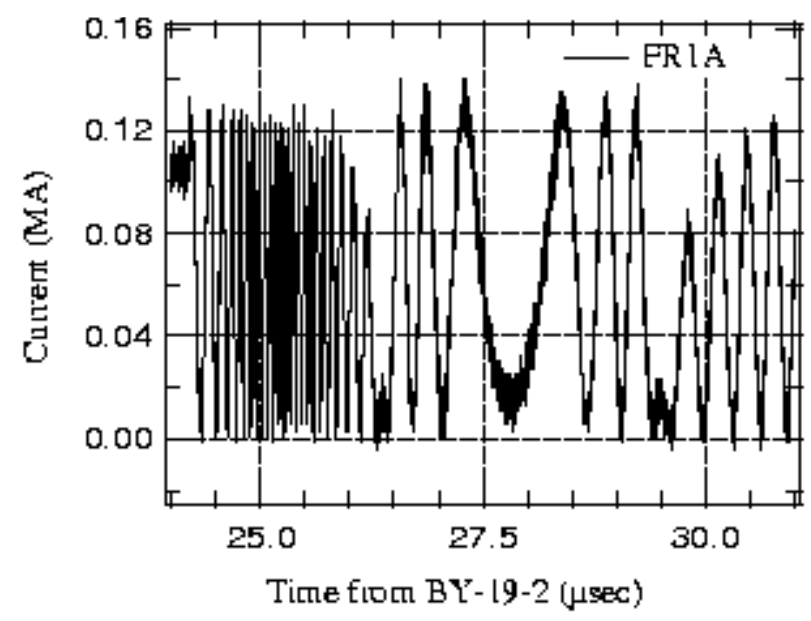

Figure 4. Raw Faraday rotation data for loop 1. Only one of the two quadrature signals is shown. 
Laser wavelengths were $835 \mathrm{~nm}$ for FR1 and $837 \mathrm{~nm}$ for FR2. The splitters were located a few meters from the ALT-1 device. Detectors and lasers were located in the bunker, connected to the loops and splitters with fibers.

Raw data from one of the Faraday rotation channels is shown in Fig. 4. The oscillations are "fringes" that relate to current through the Verdet constant. In this case, the Verdet constant is $1.19 \mathrm{MA} /$ fringe at $826 \mathrm{~nm}$. Two signals from each loop were recorded with the quadrature analysis system. In the figure it can be seen that clear data were obtained from the Faradays.

Figure 5 presents the unfolded current data from each of the four Faraday detectors overlaid on a single plot. Clearly, there is excellent agreement between all of the channels. Peak current is 32.3 MA.

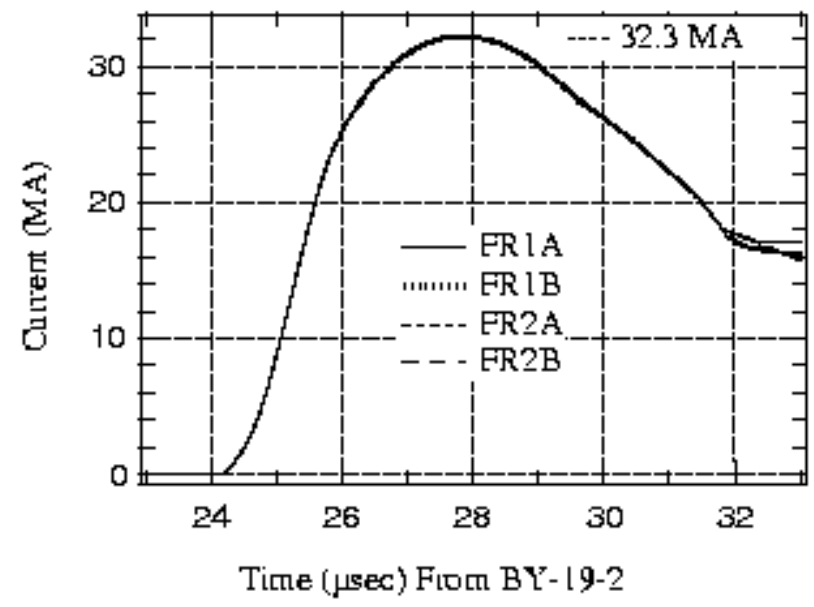

Figure 5. Analyzed Faraday rotation data. Data are unfolded from each of the two quadrature channels for each loop. Wavelength corrections are included. Peak current is 32.3 MA.

Sixteen fiber optic impact pins were located in the lower half of the CMU. Fiber optic pins are simply a 100 micron core PolyMicro synthetic silica fiber oriented radially with the end at a specific radius, and covered with a 25 micron thick aluminum foil. The foil prevents ambient light from entering the fibers. When struck by the fast moving liner, shock induced heating in the fiber produces light. Quasi-logarithmic photomultiplier tubes detect the light, and the signal is recorded on digitizers.

The pins were arranged in two axial rows of six pins each, separated azimuthally by 180 degrees. Each row started $1 \mathrm{~mm}$ from the CMU centerline, and the pins were separated by $3 \mathrm{~mm}$. An azimuthal array of pins was located $7 \mathrm{~mm}$ from the centerline. All pin tips were at 9.5 $\mathrm{mm}$ radius.

The azimuthal array consisted of four additional pins that were positioned between the axial rows. When combined with one axial pin from each row, an azimuthal array of six pins, spaced at 60 degrees, was formed. Unfortunately, data from the azimuthal array were not recorded.

Ten of the twelve axial pins were successfully recorded. Figure 6 presents the raw digitizer data for those pins.

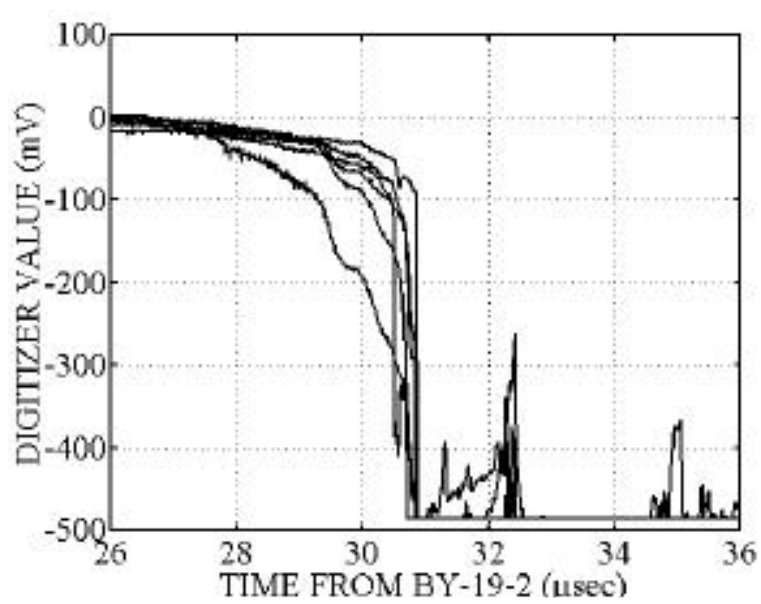

Figure 6. Raw data for fiber optic impact pins. Six of ten recorded axial pins are shown here. Slow leading edges are thought to result from light leaking into the fibers. Pin times are taken from the very sharp rising edges.

Time of arrival is taken from the sharp rising portion of the waveform. Appearance of a sharply rising portion of the waveform is an indicator of a clean, unperturbed, liner. Data from other experiments, performed with high quality radiography, have shown that the presence of instabilities on the liner surface will cause the pins to indicate no well-defined arrival time.

Figure 7 is a plot of the time-of-arrival for the ten pins recorded. Pins located at $16 \mathrm{~mm}$ are very close to the glide plane, and may indicate early arrival due to the hot plasma and metallic distortion near the sliding contact of liner with the glide plane. The remaining pins indicate the liner was very uniform. Maximum displacement from uniform arrival times is about $100 \mathrm{~ns}$, which transforms to $1.2 \mathrm{~mm}$ spatially.

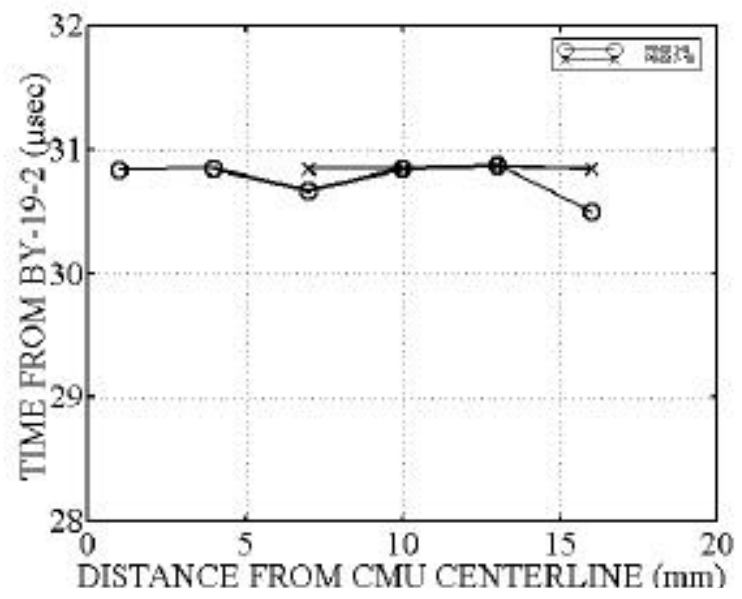

Figure 7. Time of arrival of the liner at the fiber optic pin tips vs. axial distance from the longitudinal center of the central measuring unit (CMU). 


\section{SUMMARY}

An explosive driven imploding-liner experiment was successfully fielded in Sarov, Russia. The purpose of the experiment was to observe the behavior of an imploding liner at Atlas-like parameters. A team from Los Alamos National Laboratory brought a diagnostic package to the experimental site. Three major diagnostics were VISAR to measure liner inner-surface velocity, Faraday rotation systems to measure liner current, and fiber optic pins to measure liner symmetry upon arrival at the diagnostic package. Maximum liner velocity was greater than 12 $\mathrm{km} / \mathrm{sec}$, maximum liner current was $32.3 \mathrm{MA}$, and the pins indicated that the liner was stable and axially symmetric upon arrival at the diagnostic package.

\section{ACKNOWLEDGMENTS}

The authors wish to express deep gratitude to W. Anderson, E. Armijo, J. Bartos, T. Pierce, and B. Randolph of the Los Alamos National Laboratory Target Fabrication Facility for their part in manufacturing the diagnostic assembly.

\section{REFERENCES}

[1] D. W. Scudder, S. A. Archuleta, E. O. Ballard, G. W. Barr, et al., "Atlas - a new pulsed power tool at Los Alamos," Proc. PPPS-2001 IEEE Conf. on Pulsed Power and Plasma Science, June 2001.

[2] G. A. Wurden, H. A. Davis, A. Taylor, D. Bowman, E. Ballard, S. Ney, D. Scudder, and J. Trainor, "Atlas chamber, power flow channel, and diagnostic interface design," in Proc. $11^{\text {th }}$ IEEE International Pulsed Power Conference, vol. II, 1997, pp 1291-1296.

[3] I. R. Lindemuth, W. L. Atchison, R. J. Faehl, R. E Reinovsky, "The magnetically driven imploding liner parameter space of the Atlas capacitor bank," Proc. PPPS2001 IEEE Conf. on Pulsed Power and Plasma Science, June 2001.

[4] A. M. Buyko, V. K. Chernyshev,, G. G. Ivanova, Y. N. Gorbachev, et al., "Simulation of parameters of the stationary facility "ATLAS" by means of disk EMG," Proc. PPPS-2001 IEEE Conf. on Pulsed Power and Plasma Science, June 2001.

[5] V. K. Chernyshev, A. I. Kuzyaev, A. A. Petrukhin, V. A. Vasyukov, V. V. Chernyshev, "Film protection in the radiographic experiments studying the liner compressed by magnetic field from disk EMG with HE mass of 100Kg," Proc. PPPS-2001 IEEE Conf. on Pulsed Power and Plasma Science, June 2001.
[6] W. F. Hemsing, "Velocity Sensing Interferometer (VISAR) Modification," Rev. Sci. Inst., vol. 50 1979, pp.73-78.

[7] W. L. Atchison, R. J. Faehl, I. Lindemuth, "Implications of recent improvements in conductivity models on liner, wire, and fuse design," Proc. PPPS-2001 IEEE Conf. on Pulsed Power and Plasma Science, June 2001.

[8] L.R. Veeser, G. I. Chandler, and G. W. Day, "Fiber optic sensing of pulsed currents," Photonic: High Bandwidth Applications, James Chang, ed., (SPIE 648. Bellingham, WA, 1986).p.197

[9] Stokes, J. L., et al., "Precision Current Measurements on Pegasus II Using Faraday Rotation," in Tenth IEEE International Pulsed Power Conference (Albuquerque, NM), edited by W. Baker and G. Cooperstein, IEEE, Piscataway, NJ, 1995, Vol. 1, pp. 378-383 